research. It makes fascinating reading. In the foreword, we are also promised detailed applications to solid-state theory, but these have failed to materialise.

Some topics are rather nicely handled. The standard treatment of potential wells and barriers is enhanced by the study of some of the numerical magnitudes of the effects. The use of integral equations and Green's functions is very clearly discussed, though the presentation is somewhat lacking in motivation. The treatment of the time-energy uncertainty relations, as is traditional in the Russian school, is good. The Periodic Table and Molecular Spectra receive enough discussion to give one a real flavour for what is involved. The treatment of Coulomb Scattering begins with the case of the Screened Coulomb Potential. This leads to a natural introduction to the concept of the atomic form factor, and also avoids the mathematical and conceptual complexities associated with the pure Coulomb force. The seemingly mysterious agreement between the quantum and classical calculations of the Rutherford formula, usually not commented upon, is explained. The derivation of the classical limit of scattering theory is a noteworthy inclusion. The treatment of symmetry properties, in particular of the group SU(2), is unusually good, in that it uses basic Lie Group methods, applicable to more general cases, and does not capitalise on the special and rather atypical properties of SU(2) itself.

On the negative side it has to be said that the description of "wave-particle duality" is clumsy and that the whole introductory chapter, which is wide ranging and far from simple, would be incomprehensible to a newcomer to the subject. Matrix algebra is discussed in detail, yet for an understanding of the $\delta$ function one must turn to Volume 1 of the course-a strange choice of priorities. The Hamilton-Jacobi equation is introduced, yet inadequately explained. There is a long discussion of $\alpha$ decay and of the sensitivity of barrier effects to the energy, yet no numerical estimate to illustrate it. The derivation of degenerate perturbation theory, always a tricky question, is quite unclear, and there is in general far too little illustration of the use of perturbation theory. A fair-sized portion of the book is devoted to Relativistic Quantum Mechanics (Dirac equation, Weak and Strong interactions, Feynman diagrams). Here one has the feeling that the reader will pick up only a very superficial idea of what is involved.

The reader will be confused, after having being led through the seemingly general proof in Section 73 that the dipole moment of an atom is zero, to learn somewhat later, and without explanation, that "As distinct from atoms, molecules may have a mean dipole moment".

The translation sometimes leaves much to be desired. "Causality" and "determinism" are confused, as are "picture" and "representation"; "measurement" is used instead of "dimension" and "rank" of a matrix for its "dimension". Some phrases are particularly outlandish: "We digress from other quantities ..."; "The trace of a scalar is equal to its quadrupoled value"; "The reaction $p+\gamma \rightarrow n+\pi^{+}$ representing the elementary act of the nuclear photoeffect was observed"; and so on.

Also the notation is occasionally nonstandard, and it is inexcusable that no effort was made, in translating the volume, to comply with Western usage. Examples are: \{\} for the commutator bracket, $\hat{R}_{\mathbf{x}}$ for the operator generating displacements along the $\mathrm{X}$ axis, and * used sometimes as hermitian conjugate and sometimes as complex conjugate.

By and large this is not an attractive book. It is neither fish nor fowl, and aside from the rather nice treatment of a few selected topics, it is hard to see to whom it may be recommended as a whole.

E. LEADER

\section{Art of Bonding}

The Shape and Structure of Molecules. By C. A. Coulson. Pp. 85. (Clarendon: Oxford; Oxford University: London, June 1973.) $£ 2.75$ boards; $£ 1.50$ paper.

THE aim of this book is "to show how a few quite simple ideas, not particularly abstruse or mathematical, have been able to rationalise a large part of structural chemistry". The fundamental concept of localised bonds, with their characteristic energies and electron distributions, is established for diatomic molecules, using both the Heitler-London and the molecular orbital approach. The concept of hybridisation leads to a discussion of polyatomic molecules, which is followed by a short survey of valence rules for elements in the main groups of the periodic table. The final chapter gives a very brief discussion of delocalised bonds.

A book of this length cannot do more than introduce so vast and subtle a subject. The understanding of bonding is an art at least as much as a science, and only experience can teach us which approximate or qualitative treatments are useful and what is the likely range of their validity-as tested, for example, by predicting both the correct shape and stability of molecules and the correct electronic distribution obtained from X-ray scattering Until one has digested the results of applying various bonding theories to known properties of a wide range of molecules and crystals, one cannot apply them with any confidence to the unknown. For this purpose a much larger book is needed, which would discuss critically not only many more applications of the theory given here, but also other treaments of bonding, such as that of Sidgwick, Powell, Nyholm and Gillespie. Recognising this, the present book ends with useful suggestions for further reading.

To sum up, this book can be recommended as an introduction to the art of bonding theory, outlining the particular approach of a master practitioner both of that art and of the art of exposition.

T. H. K. BARRON

\section{Isotopes in the World}

Stable Isotope Geochemistry. By Jochn Hoefs. (Minerals, Rocks, and Inorganic Materials: Monograph Series of Theoretical and Experimental Studies, vol. 9.) Pp. ix +140 . (Springer-Verlag: Berlin, New York, 1973.) 39 DM; $\$ 14.50$.

THIs small volume, which is essentially an up to date review of the most significant contributions to stable isotope geochemistry over the last ten years or so, is divided into three main sections. The first is an introduction which briefly covers some background material relating to isotopes in general, including isotope effects and fractionation processes, mass spectrometry, sample handling, and standards. This is followed by a more detailed discussion of isolation and preparation techniques, fractionation mechanisms, and the standards appropriate to the major elements studied in stable isotope geochemistry: $\mathrm{H}, \mathrm{C}, \mathrm{O}$ and $\mathrm{S}$. Other less studied elements such as Se, B, N, Si, $\mathrm{Cl}, \mathrm{Br}, \mathrm{Li}, \mathrm{K}, \mathrm{Mg}$ and $\mathrm{Ca}$ are briefly mentioned. Finally, in the third and major section, a discussion is given of the variations in stable isotope ratios in various types of geological materials, extraterrestrial samples, the water cycle, and in the atmosphere and biosphere.

Overall the text is easy to follow, largely as a result of the crisp style and of the economy of words, which allow the significant contributions in a comprehensive bibliography of about 500 references to be covered in a relatively short space

There are only a few typographical errors, for example in the equation on page 35 . My only other criticism is that the author has omitted to mention one very active area in stable isotope geochemistry: the lunar rare gas isotope studies which have contributed much to the understanding of the dynamic processes which have occurred on the Moon's surface.

This book should provide invaluable reference work for many who teach and do research in geochemistry.

J. R. Maxwell 